\title{
ANALYSIS OF ALIGNED MAGNETIC FIELD IN CASSON FLUID PAST A SHRINKING SHEET
}

\author{
Renu Devi ${ }^{1}$, Vikas Poply ${ }^{2}$, Vinita ${ }^{3}$, Manimala ${ }^{4}$ \\ E-Mail Id: ${ }^{1}$ renu15dahiya@gmail.com, ${ }^{2}$ vikaspoply@gmail.com, ${ }^{3}$ vini252011makkar@gmail.com \\ ${ }^{4}$ manimala@ansaluniversity.edu.in \\ ${ }^{1,4}$ Department of Mathematics, Ansal University, Gurgaon, Haryana India \\ ${ }^{2}$ Department of Mathematics, KLP College, Rewari, Haryana, India \\ ${ }^{3}$ Department of Mathematics, SoBAS, GD Goenka University, Gurugram, Haryana, India
}

\begin{abstract}
This article deliberates the impact of aligned MHD flow in a Casson fluid on heat and flow transfer. The influence of various fluid parameter like Casson fluid parameter, magnetic parameter, Prandtl number, aligned angle parameter. The governing partial differential equations produced the heat and flow transportaion are moulded to ordinary differential equations by considering similarity transformations. The scientific results of the differential equations have been figure out by the Runge-Kutta Fehlberg rule with the help of shooting technique. The diversity in behavior of emerging parameters characterized graphically and their results have been discussed through table.

Keywords: Casson fluid, Aligned MHD, Shrinking sheet, Boundary layer.
\end{abstract}

\section{INTRODUCTION}

Stretching sheet problems under the influence of boundary layer flow becomes a part of attraction for many researchers due to its mathematical simplicity. On the other hand, there is vital application of stretching sheet in industries as well as in engineering areas like metal spinning, polymer processing and drawing of plastic film. [1]-[4] shows the effect of boundary layer flow over stretching sheet.

During research stretching sheet becomes part of study from many years ago, after that shrinking sheet takes a concern form many researchers. Literature for flow towards shrinking sheet is limited as comparative to stretching sheet. Still after this limitation there are many applications of flow over shrinking sheet such that polymer sheets, manufacturing of filaments, glass-fiber and paper production whereas wide area of application chemical engineering and manufacturing industries also considered. Miklavcic and Wang [5] investigates characteristics of flow over shrinking sheet with suction effects while Fang and Zhong [6] discussed the effect of boundary layer flow with arbitrary velocity flow. Khan et al. [7] analyzed the heat transfer effects on horizontal stretching/shrinking sheet.

Stagnation flow exits due to its importance in boundary layer flow. Problem is observed in case of stretching and shrinking balloon. It is noticed that solutions does not exists due to boundary layer flow, so after adding the effect of stagnation flow on boundary layer make possibility of similarity solution that gives exact results. Wang [8] considered the effects of stagnation flow over shrinking sheet whereas [9]-[12] all concern with heat transfer analysis of boundary layer flow with stagnation point over stretching/shrinking sheet. Mahapatra and Nandy [13] taken unsteady stagnation point flow whereas Lok and Pop [14] explained about unsteady separated stagnation point. On the other hand, Mahapatra et al. [15] described oblique flow. Rosali et al. [16] further investigates their study in porous medium. Zaimi and Ishak [17] used permeable stretching/shrinking sheet.

Magnetohydrodynamic (MHD) is that term in which there is existence of magnetic field on electrically conducting moving fluid. Its applications make this area special in research field for example electromagnetic pump, designing of heat exchanger, accelerator and generator. It was observed that magnetic field strength affect the viscous fluid flow as discussed by [18]-[22]. Study of stagnation point with MHD fluid flow properties done by [23] while an important part of flow which is oblique MHD flow was expanded by Lok et al. [24]. Problems related to MHD boundary layer were investigated by [25] and [26]. Bhattacharya and Krishnendu [27] performed the heat transfer and MHD flow with radiation effect in presence of heat source/sink and suction/injection. Chauhan and Agrawal [28] extended their study for shrinking sheet as well as porous substrate plate.

Casson fluid is a category of non-Newtonian fluid which has properties of shear thinning fluid that is performed by yield stress and makes the fluid flow possible otherwise fluid seems like a solid. Sauce, jelly, soup and honey are some examples of viscous fluids that are added in casson fluid whereas human blood is most appropriate example of Casson fluid which is main part of bio-medical field. Therefore, Sheikh and Abbas [29] includes Casson fluid to solve heterogeneous and homogeneous solutions.

Up to our knowledge, no study has been carried out so far to study; the flow and heat transportation on Casson fluid along with aligned MHD past a shrinking sheet. The motive of current assessment is to analyze the aligned MHD effects on Casson fluid.

\section{MATERIALS AND METHODS}

Steady 2D Casson fluid flow of a non-compressible, viscous, electrical conducting fluid on a shrinking sheet is considered. Aligned magnetic field are also assumed the impact on fluid flow. $u_{W}(x)$ and $T_{W}$ are the linear velocity and uniform temperature on shrinking surface respectively (as shown in Fig. 2.1). 


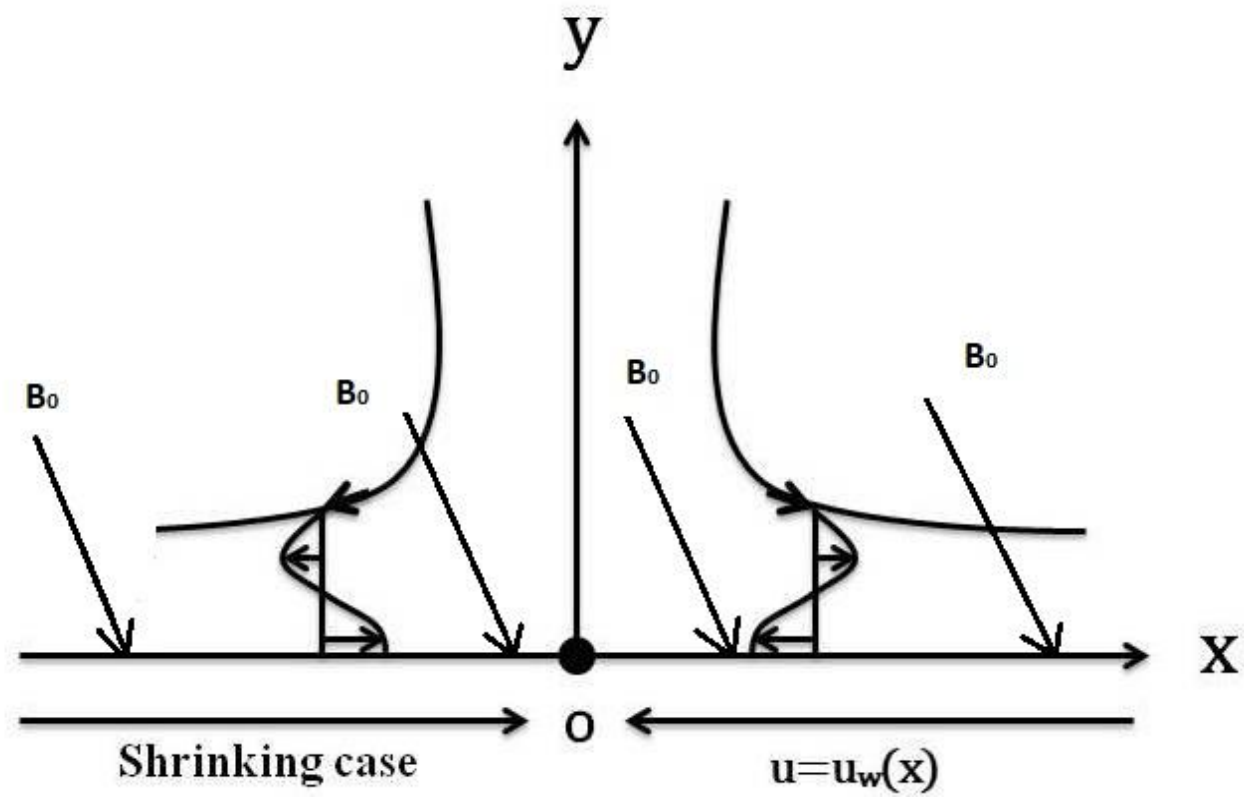

Fig. 2.1 Schematic Diagram

The generating equations of flow under the above assumptions are described as:

$\frac{\partial \mathrm{u}}{\partial \mathrm{x}}+\frac{\partial \mathrm{v}}{\partial \mathrm{y}}=0$

$\mathrm{u} \frac{\partial \mathrm{u}}{\partial \mathrm{x}}+\mathrm{v} \frac{\partial \mathrm{u}}{\partial \mathrm{y}}=\mathrm{v}\left(1+\frac{1}{\beta}\right)\left(\frac{\partial^{2} \mathrm{u}}{\partial \mathrm{x}^{2}}+\frac{\partial^{2} \mathrm{u}}{\partial \mathrm{y}^{2}}\right)-\frac{\sigma \mathrm{B}_{0}^{2} \mathrm{u} \sin ^{2} \mathrm{l}}{\rho}$

$\rho \mathrm{C}_{\mathrm{P}}\left(\mathrm{u} \frac{\partial \mathrm{T}}{\partial \mathrm{x}}+\mathrm{v} \frac{\partial \mathrm{T}}{\partial \mathrm{y}}\right)=\mathrm{K} \frac{\partial^{2} \mathrm{~T}}{\partial \mathrm{y}^{2}}$

where velocity along $y$ (vertical axis) and $x$ (horizontal axis) - axes are taken as $v$ and $u$ respectively. $v, \sigma, \mathrm{C}_{\mathrm{P}}, T, K$ and $B_{0}$ denotes the kinematic viscosity, electrical conductivity, specific heat at constant pressure, fluid temperature, thermal conductivity and magnetic field strength of the fluid respectively.

Restrictions on the boundary are describing the flow model as:

$$
\left.\begin{array}{ccc}
\mathrm{y}=0 & \mathrm{u}=\mathrm{u}_{\mathrm{w}}(\mathrm{x})=-\mathrm{bx}, & \mathrm{v}=0, \\
\mathrm{y} \rightarrow \infty & \mathrm{T}=\mathrm{T}_{\mathrm{w}} \\
\mathrm{u}=0, & \mathrm{~T}=\mathrm{T}_{\infty}
\end{array}\right\}
$$

Where $\mathrm{b}$ is non-negative invariable values of dimension $\left(\right.$ time $\left.^{-1}\right)$. The fluid having unvarying temperature $T_{\infty}$ very far from the surface.

Introducing $\xi=\sqrt{\frac{b}{v}} x, \eta=\sqrt{\frac{b}{v}} y$ and $\psi(\xi, \eta)$ (stream function) as dimensionless variables such that $u=\frac{\partial \psi}{\partial \eta}$ and $v=-\frac{\partial \psi}{\partial \xi}$. The Boundary condition in term of stream function $\psi(\xi, \eta)$ is given by

We required solution of equation (6) from the relation $\psi=\xi f_{a}(\eta)$, where $f_{a}(\eta)$ are referred as tangential and normal parts of flow. Also, $v=-f_{a}(\eta)$ and $u=\xi f_{a}{ }^{\prime}(\eta)$.

Equation (1) is contented by given $v$ and $u$ and equation (2) and (4) transformed to equation (5) and (6),

$\left(1+\frac{1}{\beta}\right) f_{a}{ }^{\prime \prime \prime}(\eta)+f_{a}(\eta) f_{a}{ }^{\prime \prime}(\eta)-\left(f_{a}{ }^{\prime}(\eta)\right)^{2}-M \sin ^{2} l f_{a}{ }^{\prime}(\eta)=0$

$\mathrm{f}_{\mathrm{a}}(0)=0, \mathrm{f}_{\mathrm{a}}{ }^{\prime}(0)=-1, \mathrm{f}_{\mathrm{a}}{ }^{\prime}(\infty)=0$

Here $M=\frac{\sigma B_{0}^{2}}{b \rho}$ is the Chandershekhar number (magnetic parameter)

Dimensionless temperature $\theta(\eta)=\left(T-T_{\infty}\right) /\left(T_{w}-T_{\infty}\right)$. Substituting $\theta(\eta)$ in equation (3), we get

$\theta^{\prime \prime}(\eta)+\operatorname{Pr} \theta^{\prime}(\eta) f_{a}(\eta)+\operatorname{Pr} \theta(\eta)=0$

where, $\operatorname{Pr}\left(=\mu \mathrm{C}_{\mathrm{P}} / \mathrm{K}\right)$.

Corresponding boundary conditions of (4) reduces to $\theta(0)=1, \theta(\infty)=0$

The terms of practical importance are $C_{f}$ and $N u_{x}$ are defined as

The wall shear stress $\tau_{w}$ is described as $\mathrm{C}_{\mathrm{f}}=\frac{\tau_{\mathrm{w}}}{\rho\left(\mathrm{u}_{\mathrm{w}}\right)^{2}}$ then $\mathrm{C}_{\mathrm{f}} \propto f_{a}^{\prime \prime}(0)$ when $\tau_{\mathrm{w}}=\left.\mu\left(1+\frac{1}{\beta}\right) \frac{\partial u}{\partial y}\right|_{\mathrm{y}=0}$

The wall heat flux $q_{w}$ is given as $N u_{x}=\frac{x q_{w}}{K\left(T_{w}-T_{\infty}\right)}$ then $N u_{x} \propto-\theta^{\prime}(0)$ when $q_{w}=-\left.\left(K \frac{\partial T}{\partial y}\right)\right|_{y=0}$

DOI Number: https://doi.org/10.30780/specialissue-ICACCG2020/0036

Paper Id: IJTRS-ICACCG2020-036 
ICACCG2020 30-31 July, 2020, Ansal University, Gurgaon, India

International Journal of Technical Research \& Science (Special Issue)

\section{RESULT AND DISCUSSION}

Here, Runge-Kutta Fehlberg technique is considered to find out the solution of differential equations (5) and (7) with the help of shooting procedure. Velocity and dimensionless temperature of the model have been acquired for distinct entries of various fluid parameters. The value of $\mathrm{C}_{\mathrm{f}} \propto f_{a}^{\prime \prime}(0)$ and $N u_{x} \propto-\theta^{\prime}(0)$ are computed for further analysis.

Fig. 3.1 exhibits the significance of velocity profile under influence of $\beta$. Figure 3.1 expresses that the fluid velocity rise with rising entries of $\beta$. This graph elaborates that enhancement in the value of $\beta$ will decline yield stress that hurdles the free movement of fluid particles and hence boundary layer thickness reduced. Figure 3.2 disclosures the behavior of $\beta$ for temperature distribution. Figure 3.2 indicates that increases, fluid temperature reduced and this graph elaborates that enhancement in the value of $\beta$ enhances fluid velocity that will intensify the heat transfer. We notice from the Table 3.1 that with the increase in Casson fluid parameter $\beta, C_{\mathrm{f}} \propto f_{a}^{\prime \prime}(0)$ and $N u_{x} \propto-\theta^{\prime}(0)$ values rises.

Table-3.1 Outcomes of $f_{a}^{\prime \prime}(0)$ and $-\theta^{\prime}(0)$ for fixed entries of $M=10, l=\pi / 3$ and $P r=1.1$

\begin{tabular}{|c|c|c|}
\hline $\boldsymbol{\beta}$ & $\boldsymbol{f}_{\boldsymbol{a}}^{\prime \prime}(\mathbf{0})$ & $-\boldsymbol{\theta}^{\prime}(\mathbf{0})$ \\
\hline $\mathbf{2}$ & 2.08237 & -0.85646 \\
\hline $\mathbf{4}$ & 2.28068 & -0.83984 \\
\hline $\mathbf{8}$ & 2.40390 & -0.83040 \\
\hline
\end{tabular}

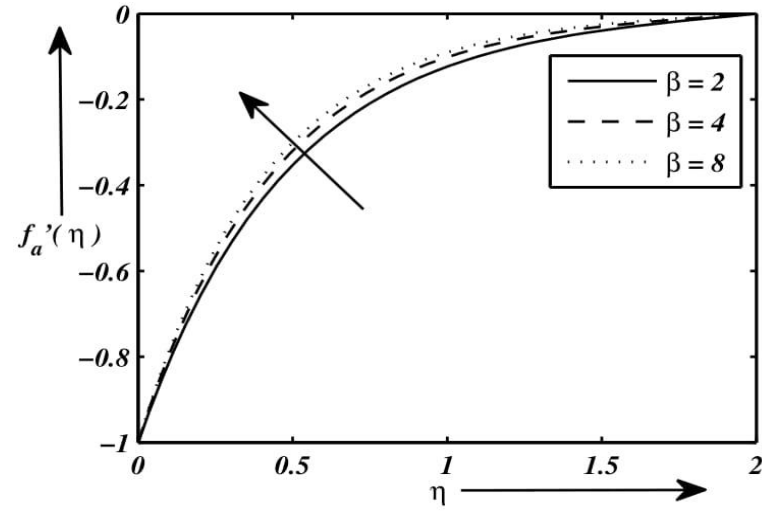

Fig. 3.1 Pattern of $f_{a}{ }^{\prime}(\eta)$ for distinct $\beta$ with fixed entries $M=10, l=\pi / 3, P r=1.1$

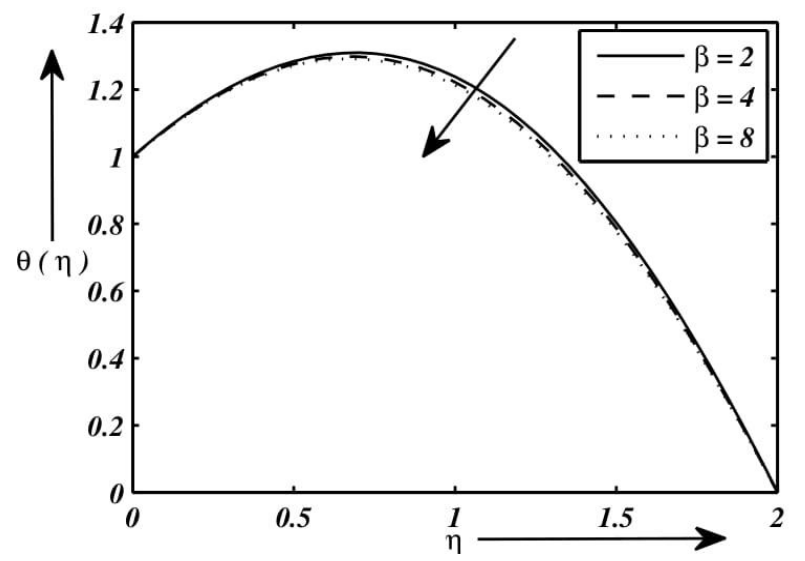

Fig. 3.2 Pattern of $\theta(\eta)$ for distinct $\beta$ with fixed entries $M=10, l=\pi / 3, \operatorname{Pr}=1.1$

Fig. 3.3 manifests variation of fluid velocity against $M$ on velocity. This figure shows that existence of magnetic parameter $M$ resist the fluid particles to move freely and main reason behind the resistance is that $M$ produces Lorentz force and this magnetism behavior can be adopted for controlling the fluid movement. Thus an enhancement in $M$ causes the enhancement of velocity distribution as depicted via Figure 3.3. Figure 3.4 represents that the temperature profile declines marginally with rise in $M$ because a slight conversion in $N u_{x}$ has been seen with rising $M$ (shown in Table 3.2). As heat transfer rate can control by the help of magnetism. Therefore, it can conclude that in flow characteristics, $M$ plays a substantial role. From Table 3.2, we observed that with the increase in magnetic parameter $\mathrm{M}, \mathrm{C}_{\mathrm{f}} \propto f_{a}^{\prime \prime}(0)$ and $N u_{x} \propto-\theta^{\prime}(0)$ both are increases.

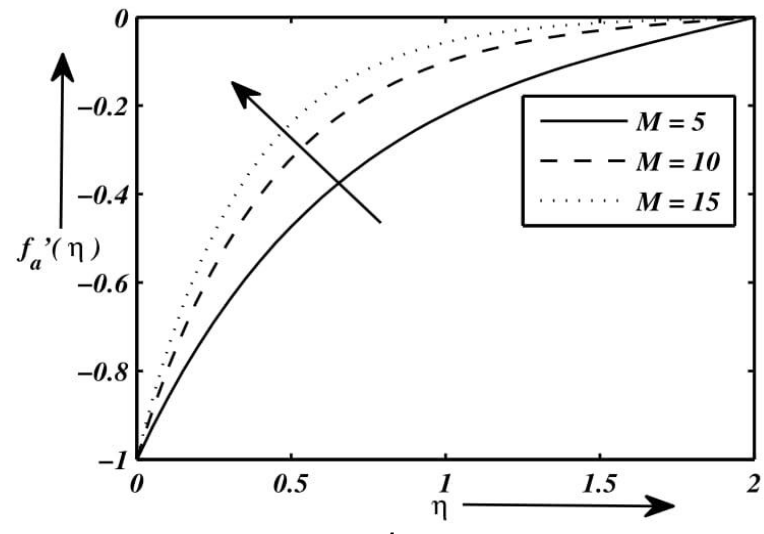

Fig. 3.3 Pattern of $f_{a}{ }^{\prime}(\eta)$ for distinct $M$ with

Fig. 3.4 Pattern of $\theta(\eta)$ for distinct $M$ with

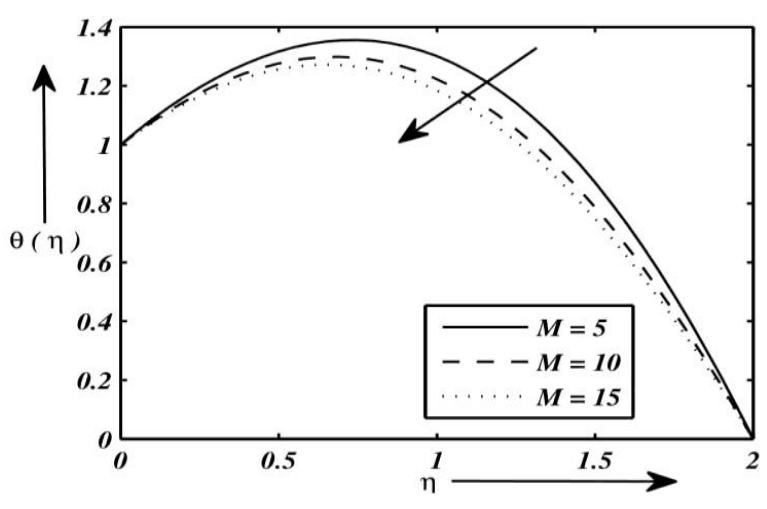

fixed entries $\beta=4, l=\pi / 3, \operatorname{Pr}=1.1$

fixed entries $\beta=4, l=\pi / 3, \operatorname{Pr}=1.1$ 
ICACCG2020 30-31 July, 2020, Ansal University, Gurgaon, India

International Journal of Technical Research \& Science (Special Issue)

ISSN No.:2454-2024 (online)

Table-3.2 Outcomes of $f_{a}^{\prime \prime}(0)$ and $-\theta^{\prime}(0)$ for fixed entries of $\beta=4, l=\pi / 3$ and $\operatorname{Pr}=1.1$

\begin{tabular}{|c|c|c|}
\hline $\boldsymbol{M}$ & $\boldsymbol{f}_{\boldsymbol{a}}^{\prime \prime}(\mathbf{0})$ & $-\boldsymbol{\theta}^{\prime}(\mathbf{0})$ \\
\hline $\mathbf{5}$ & 1.48831 & -0.91941 \\
\hline $\mathbf{1 0}$ & 2.28068 & -0.83984 \\
\hline $\mathbf{1 5}$ & 2.86360 & -0.80034 \\
\hline
\end{tabular}

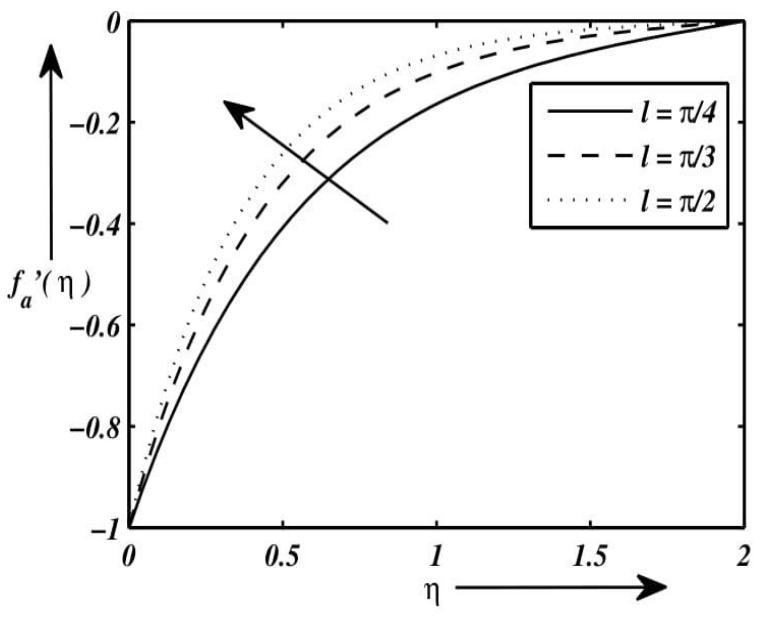

Fig. 3.5 Pattern of $f_{a}{ }^{\prime}(\eta)$ for distinct $l$ with fixed entries $\beta=4, M=10, \operatorname{Pr}=1.1$

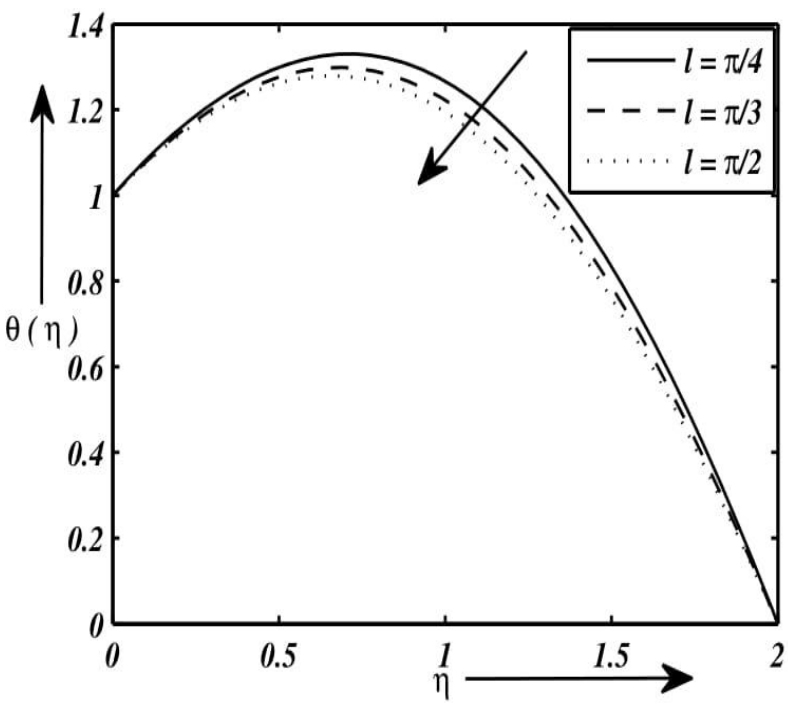

Fig. 3.6 Pattern of $\theta(\eta)$ for distinct $l$ with fixed entries $\beta=4, M=10, \operatorname{Pr}=1.1$

Fig. 3.5 examine to analyze the impact of $l$ on dimensionless velocity distribution. Here, increment in velocity profile has been noticed with the rising in value of $l$. This happened because of higher value of $l$ that reinforces the applied magnetic field and this situation create reverse force to flow. That reverse force reduced the velocity which exhibited by Fig. 3.5. This force has capacity to reduce boundary layer thickness. In Fig. 3.6, the effect of $l$ on temperature distribution is observed. As $l$ increases temperature of fluid and thermal boundary thickness both reduced. From Table 3.3, we observed that with the increase in $l$ then $\mathrm{C}_{\mathrm{f}} \propto f_{a}^{\prime \prime}(0)$ and $N u_{x} \propto-\theta^{\prime}(0)$ both are increases.

Table-3.3 Outcomes of $f_{a}^{\prime \prime}(0)$ and $-\theta^{\prime}(0)$ for fixed entries of $\beta=4, l=\pi / 3$ and $\operatorname{Pr}=1.1$

\begin{tabular}{|c|c|c|}
\hline $\boldsymbol{l}$ & $\boldsymbol{f}_{\boldsymbol{a}}^{\prime \prime}(\mathbf{0})$ & $-\boldsymbol{\theta}^{\prime}(\mathbf{0})$ \\
\hline $\boldsymbol{\pi} / \mathbf{4}$ & & -0.88472 \\
\hline $\boldsymbol{\pi} / \mathbf{3}$ & 1.79066 & -0.83984 \\
\hline $\boldsymbol{\pi} / \mathbf{2}$ & 2.28068 & -0.81124 \\
\hline
\end{tabular}

\section{CONCLUSION}

Steady 2D Casson fluid flow of a non-compressible, viscous, orthogonally electrical conducting fluid on a shrinking sheet with has been studied. Runge-Kutta Fehlberg has been used to solve numerically the differential equations with their corresponding boundary conditions through shooting technique. The numerical results obtained in this study include the influence of aligned magnetic field and Casson parameter on temperature and velocity profiles of the flow. This analysis revealed major recommendations of the outcomes are compiled as below:

$>$ Fluid velocity increases and momentum boundary layer thickness decreases with an increase in Casson parameter $\beta$, magnetic parameter $M$ and aligned angle parameter $l$.

$>$ Fluid temperature and thermal boundary layer thickness both decreases as we increase in Casson parameter $\beta$, magnetic parameter $M$ and aligned angle parameter $l$.

These results have possible technological applications in liquid-based systems involving shrinking materials. The finding of this study may serve as to control the rate of heat transportation. A future study of this analysis can be done by considering stretching cylindrical surfaces. 
ICACCG2020 30-31 July, 2020, Ansal University, Gurgaon, India

International Journal of Technical Research \& Science (Special Issue)

ISSN No.:2454-2024 (online)

\section{NOMENCLATURE}

\begin{tabular}{|c|c|c|c|}
\hline$R$ & constant & $f_{a}$ & normal component of flow \\
\hline$\beta$ & Casson parameter & $P r$ & Prandtl number \\
\hline$x, y$ & cartesian coordinates & $C_{P}$ & specific heat \\
\hline$T$ & dimensionaless temperature & $\Psi$ & skin friction coefficient \\
\hline$\sigma$ & electrical conductivity & $C_{f}$ & thermal counductivity \\
\hline$\rho$ & fluid density & $T_{w}$ & temperature at surface \\
\hline$v$ & kinematic viscosity & $T$ & temperature profile \\
\hline$q_{m}$ & local heat flux & $T_{\infty}$ & uniform ambient temperature \\
\hline$N u_{x}$ & local Nusselt number & $u, v$ & velocity component \\
\hline$M$ & magnetic parameter (Chandershekhar & & number) \\
\hline$B_{0}$ & magnetic field strength & $\tau_{\mathrm{w}}$ & wall shear stress \\
\hline
\end{tabular}

\section{REFERENCES}

[1] Mahapatra T. R., \& Gupta A. S. (2008). Stagnation-point Flow towards a Stretching Surface. The Canadian Journal of Chemical Engineering, 81, 258-263.

[2] Mustafa M., Hayat T., Ioan P., \& Hendi A. (2012). Stagnation-Point Flow and Heat Transfer of a Casson Fluid towards a Stretching Sheet. Zeitschrift fr Naturforschung A, 67, 70-76.

[3] Lok Y., Amin N., \& Pop I. (2006). Non-orthogonal stagnation point flow towards a stretching sheet. International Journal of Non-Linear Mechanics, 41, 622-627.

[4] Singh P., Tomer N. S., Kumar S., \& Sinha D. (2010). MHD oblique stagnation-point flow towards a stretching sheet with heat transfer. International Journal of Applied Math and Mechanics, 6(13), 94-111.

[5] Miklavi M., \& Wang C. Y. (2006). Viscous flow due to a shrinking sheet. Quarterly of Applied Mathematics, 64, 283-290.

[6] Fang T., \& Zhong Y. (2010). Viscous flow over a shrinking sheet with an arbitrary surface velocity. Communications in Nonlinear Science and Numerical Simulation, 15, 3768-3776.

[7] Khan Y., Faraz Q.Wu, N., \& Yildirim A. (2011). The effects of variable viscosity and thermal conductivity on a thin film flow over a shrinking/stretching sheet. Computers \& Mathematics with Applications, 61, 3391-3399.

[8] Wang C. (2008). Stagnation flow towards a shrinking sheet. International Journal of Non-Linear Mechanics, 43, 377-382.

[9] Bachok N., Ishak A., \& Pop I. (2010). Melting heat transfer in boundary layer stagnation-point flow towards a stretching/shrinking sheet. Physics Letters A, 374, 4075-4079.

[10] Bachok N., Ishak A., \& Pop I. (2013). Stagnation point flow toward a stretching/shrinking sheet with a convective surface boundary condition. Journal of the Franklin Institute, 350, 2736-2744.

[11] Bhattacharyya K., Mukhopadhyay S., \& Layek G. (2011). Slip effects on boundary layer stagnation-point flow and heat transfer towards a shrinking sheet. International Journal of Heat and Mass Transfer, 54, 308-313.

[12] Bhattacharyya K. (2013). Heat transfer analysis in unsteady boundary layer stagnation-point flow towards a shrinking/stretching sheet. Ain Shams Engineering Journal, 4, 259-264.

[13] Mahapatra T. R., \& Nandy S. K. (2013). Slip effects on unsteady stagnation-point flow and heat transfer over a shrinking sheet. Meccanica, 48, 1599-1606.

[14] Lok Y. Y., \& Pop I. (2014). Stretching or shrinking sheet problem for unsteady separated stagnation-point flow. Meccanica, 49, 1479-1492.

[15] Mahapatra T. R., Nandy S. K, \& Gupta A. S. (2012). Oblique stagnation-point flow and heat transfer towards a shrinking sheet with thermal radiation. Meccanica, 47, 1325-1335.

[16] Rosali H., Ishak A., \& Pop I. (2011). Stagnation point flow and heat transfer over a stretching/shrinking sheet in a porous medium. International Communications in Heat and Mass Transfer, 38, 1029-1032.

[17] Zaimi K., \& Ishak A. (2015). Boundary layer flow and heat transfer over a permeable stretching/shrinking sheet with a convective boundary condition. Journal of Applied Fluid Mechanics, 8(3), 499-505. 
[18] Abbasbandy S., \& Roohani Ghehsareh H. (2013). Solutions for MHD viscous flow due to a shrinking sheet by HankelPad method. International Journal of Numerical Methods for Heat \& Fluid Flow, 23, 388-400.

[19] Akyildiz F. T., \& Siginer D. A. (2010). Existence results and numerical simulation of magnetohydrodynamic viscous flow over a shrinking sheet with suction. Mathematical and Computer Modelling, 52, 346-354.

[20] Van Gorder R. A., Vajravelu K., \& Pop I. (2012). Hydromagnetic stagnation point flow of a viscous fluid over a stretching or shrinking sheet. Meccanica, 47, 31-50.

[21] Noor N., Awang Kechil S., \& Hashim I. (2010). Simple non-perturbative solution for MHD viscous flow due to a shrinking sheet. Communications in Nonlinear Science and Numerical Simulation, 15, 144-148.

[22] Fang T., \& Zhang J. (2009). Closed-form exact solutions of MHD viscous flow over a shrinking sheet. Communications in Nonlinear Science and Numerical Simulation, 14, 2853-2857.

[23] Aman F., Ishak A., \& Pop I. (2013). Magnetohydrodynamic stagnation-point flow towards a stretching/shrinking sheet with slip effects. International Communications in Heat and Mass Transfer, 47, 6872.

[24] Lok Y. Y., Merkin J. H., \& Pop I. (2015). MHD oblique stagnation-point flow towards a stretching/shrinking surface. Meccanica. 50, 2949-2961.

[25] Cortell R. (2010). On a certain boundary value problem arising in shrinking sheet flows. Applied Mathematics and Computation, 217, 4086-4093.

[26] Merkin J., \& Kumaran V. (2010). The unsteady MHD boundary-layer flow on a shrinking sheet. European Journal of Mechanics - B/Fluids, 29, 357-363.

[27] Bhattacharyya K. (2011). Effects of radiation and heat source/sink on unsteady MHD boundary layer flow and heat transfer over a shrinking sheet with suction/injection. Frontiers of Chemical Science and Engineering, 5, 376-384.

[28] Chauhan D. S., \& Agrawal R. (2011). MHD flow and heat transfer in a channel bounded by a shrinking sheet and a plate with a porous substrate. Journal of Engineering Physics and Thermophysics, 84, 1034-1046.

[29] Sheikh M., \& Abbas Z. (2015). Homogeneous and heterogeneous reactions in stagnation point flow of Casson fluid due to a stretching/shrinking sheet with uniform suction and slip effects. Ain Shams Engineering Journal.

[30] Poply, V., Singh, P., \& Yadav, A. K., (2015). A Study of Temperature-dependent Fluid Properties on MHD Free Stream Flow and Heat Transfer over a Non-Linearly Stretching Sheet, Procedia Engineering, 127, 391-397.

[31] Vinita V., \& Poply V. (2019). Impact of outer velocity MHD slip flow and heat transfer of nanofluid past a stretching cylinder. Mater. Today Proc., 26, 3429-3435, doi: 10.1016/j.matpr.2019.11.304.

[32] Devi R., Poply V., \& Manimala. (2019). Impact of inclined outer velocity in MHD Casson fluid over stretching sheet. Int. J. of Advanced Trends in Comp. Applic., 1, 32-38.

[33] Devi R., Poply V., \& Manimala. (2019). Impact of aligned MHD flow with inclined outer velocity for casson nanofluid over a stretching sheet. Heat Transfer, 1-24. 